\title{
Salvage lung surgery in long-term survivors after biological and immunotherapeutic treatments
}

\author{
Lawek Berzenji, Paul E. Van Schil \\ Department of Thoracic and Vascular Surgery, University Hospital Antwerp, Edegem, Belgium \\ Contributions: (I) Conception and design: All authors; (II) Administrative support: PE Van Schil; (III) Provision of study materials or patients: PE Van \\ Schil; (IV) Collection and assembly of data: L Berzenji; (V) Data analysis and interpretation: All authors; (VI) Manuscript writing: All authors; (VII) \\ Final approval of manuscript: All authors. \\ Correspondence to: Paul E. Van Schil, MD, PhD. Department of Thoracic and Vascular Surgery, Antwerp University Hospital, Wilrijkstraat 10, B-2650 \\ Edegem (Antwerp), Belgium. Email: paul.van.schil@uza.be.
}

\begin{abstract}
Despite increasing efforts regarding screening and early detection, the majority of new lung cancers are still diagnosed at an advanced stage. For advanced cases of non-small cell lung cancer (NSCLC), treatment is often based on systemic chemoradiotherapy (CRT). However, in the last few decades, emerging modalities such as targeted therapy and immunotherapy have altered treatment paradigms, and have vastly improved overall and disease-free survival for a large group of patients. Furthermore, tumours that were initially deemed inoperable may now demonstrate a downstaging of their cancer to operable disease status. In these patients, salvage lung resections can be considered as a treatment to obtain complete resection. However, data on patient selection criteria, optimal surgical timing, postoperative complications, and outcomes of salvage surgery in patients receiving these systemic therapies are scarce. In this review, we aim to summarize the most recent literature regarding salvage lung surgery in patients that have received targeted therapy or immunotherapy.
\end{abstract}

Keywords: Salvage surgery; non-small cell lung cancer (NSCLC); targeted therapy; immunotherapy; outcomes

Received: 13 May 2020; Accepted: 20 June 2020; Published: 10 July 2021.

doi: 10.21037/shc-2019-rpts-25

View this article at: http://dx.doi.org/10.21037/shc-2019-rpts-25

\section{Introduction}

Lung cancer is worldwide the most common malignancy and remains one of the leading causes of mortality $(1,2)$. Despite the fact that rates of lung cancer vary around the world, an overall increase in new cases of non-small cell lung cancer (NSCLC) are seen, mainly due to rising incidences in developing countries (3). Recent estimates have shown a global incidence rate of 1.8 million lung cancer diagnoses, comprising $13 \%$ of all new global cancer diagnoses (4). Furthermore, the 5-year survival rate of lung cancer is around $18 \%$, a significantly lower long-term survival rate compared to any of the other leading cancers (5). This low survival rate is mainly due to the fact that the vast majority of lung cancer patients are diagnosed with advanced-stage disease (6). Treatment for NSCLC depends on the stage and extensiveness of the disease. For early stage NSCLC, lobectomy with hilar and mediastinal lymph node dissection is considered the first line of therapy $(7,8)$. However, in more advanced cases of NSCLC, surgery is often not indicated and systemic chemoradiotherapy (CRT) is considered the mainstay of treatment (9). If possible, a trimodal approach with chemotherapy, radiation therapy, and surgery is used in many cases to obtain optimal diseasefree and overall survival (DFS and OS, respectively) rates. Earlier studies using this combined approach have shown acceptable results with 5 -year OS rates ranging from $16-38 \%(10,11)$. However, despite these curative-intent treatments, approximately $39 \%$ of all patients develop locoregional recurrence or have residual tumour within 2 years after initial treatment $(12,13)$. In cases of local failure, treatment options are often limited and results 
of subsequent treatments are disappointing generally. Moreover, there is no clear consensus on which therapeutic approach would be most suitable in cases of local or regional failure (14). In recent years, a large number of targeted and immunotherapeutic treatments have been added to the therapeutic arsenal for the treatment of (advanced) NSCLC. New knowledge regarding cancer biology and cancer immune evasion have already altered treatment paradigms and vastly improved the expected outcomes of many patients with advanced stages of NSCLC (6). Despite the increasing availability of new modalities, many patients that are treated with systemic therapies for advanced stages of NSCLC will develop recurrence in time, often manifesting as distant metastases with or without local relapse (15). However, in a small proportion of these patients, the recurrence is an isolated local relapse that is not suitable for curative-intent radiotherapy. In these patients, complete resection is the only curative-intent treatment modality available. This type of surgery is referred to as 'salvage surgery' $(16,17)$. Despite the growing knowledge regarding long-term outcomes of biological and immunotherapeutic treatments, there is a lack of data concerning (salvage) surgery in this patient group. In this study, we aim to summarize the latest results on salvage surgery in patients that have received targeted therapy of immunotherapy.

\section{Treatment after local failure}

Local failure is defined as recurrence and/or residual tumour, and is the leading cause of death in patients undergoing initial curative-intent definitive CRT (18). Several treatment modalities have been proposed for patients with local failure. In some cases, high-dose reirradiation after definitive CRT may be considered. Reported 2-year overall and disease-free survival rates in this patient group are $32 \%$ and $37 \%$, respectively. Locoregional failure is present in approximately $49 \%$ of all patients at 1 -year post-treatment. In addition, treatments with highdose reirradiation are often associated with excess toxicities to surrounding organs-at-risk, including the heart, lungs, spinal cord, and oesophagus $(14,18,19)$. Previous studies investigating high-dose reirradiation after local failure have shown increased frequencies of pneumonitis, oesophagitis, and fatal bleeding risks. Furthermore, radiation therapy can significantly reduce total lung and diffusion capacity, with lung function impairment even being reported 6 months post-treatment $(14,20)$.

Second-line chemotherapy has also been described as a treatment modality after local failure and for patients with progressive disease. However, recent studies have shown only minimal survival benefits in this patient population, with median long-term survival and progressive-free survival rates of 9 and 6 months, respectively $(21,22)$. Furthermore, previous studies have shown very low response rates of only $10 \%$ to platinum-based chemotherapy for recurrent NSCLC after definitive CRT (23).

In recent years, salvage lung surgery has increased in popularity as studies have shown improved OS and DFS rates in selected patient groups (24-27). The term salvage surgery generally refers to surgical resections of persistent or recurrent primary lung tumours after previous local and/or systemic therapies have failed (28). Currently, there are no guidelines regarding specific indications for salvage surgery. In the majority of cases, this type of surgery is performed in patients with progressive disease after definitive chemo- and/or radiotherapy (29). However, other indications may be possible based on the specific evaluation of each patient (16). The clinical significance of salvage lung surgery remains controversial and comparing results of studies is often complicated due to differences in the definition of salvage lung surgery among investigators and in the patient selection of these studies (28).

Previous studies have shown that salvage lung surgery is technically feasible and has acceptable mortality and morbidity rates (23-25). Even in patients undergoing extended anatomical resections greater than a lobectomy, such as a pneumonectomy, long-term outcomes seem to be acceptable (14). Five-year overall survival rates range widely between $20-75 \%$ in earlier studies, mostly due to varying indications and results based on small patient populations $(14,25,26,30,31)$. In a recent retrospective study by Sonobe et al., 29 patients undergoing salvage resection after chemotherapy or CRT were included for further analysis. Five-year overall and recurrence-free survival were $51 \%$ and $49 \%$, respectively. Despite promising results from this study and other similar studies, there is still a lack of prospective data and results based on larger sample sizes (32).

\section{Neoadjuvant biological and immunotherapy in resectable patients}

The introduction of targeted therapies and immunotherapy has caused a paradigm shift in the treatment of NSCLC in the last few decades $(6,33)$. Therapies that target specific mutations of the epidermal growth factor receptor (EGFR) and on the abnormal fusion of the anaplastic lymphoma 
kinase (ALK), and treatments that exploit the mechanisms which tumours use to evade immune recognition-such as the use of monoclonal antibodies targeting the immune regulatory proteins cytotoxic T-lymphocyte-associated protein 4 (CTLA-4) and programmed death-ligand 1 (PDL1) - have now become viable therapeutic modalities for advanced NSCLC (33-37). With these new therapies, survival outcomes of a substantial proportion of patients with advanced NSCLC have improved immensely. Results of recent clinical trials have shown favourable treatment responses in specific subsets of patients with metastatic NSCLC (38). Despite the success of these systemic therapies, cancer surgery remains the most effective treatment strategy for resectable disease (7). However, data on the safety and feasibility of lung surgery after immunotherapy and targeted therapies is scarce.

Earlier studies have suggested that patients treated with immunotherapy have increased risks of complications such as pneumonitis, adrenal insufficiency, and thyroiditis $(39,40)$. Although the majority of these complications are not severe, the effects on the perioperative care of patients and the technical aspects of lung resections are not clear. In a retrospective study by Bott et al., 19 patients with metastatic or unresectable tumours underwent 22 lung resections (lobectomy, bilobectomy, or pneumonectomy) after therapy with anti-PD-1 agents (nivolumab and pembrolizumab), anti-CTLA-4 agents (ipilimumab), or anti-PD-L1 agents (durvalumab and atezolizumab). The most common tumours were NSCLC ( $\mathrm{n}=9)$ and melanoma $(\mathrm{n}=7)$. In 95\% of all cases, an R0 resection could be achieved. Two-year OS and DFS were $77 \%$ and $42 \%$, respectively. Postoperative complications were mostly minor; however, the authors noted that operations may be more challenging due to posttreatment adhesions (34).

In the recent CheckMate-159 trial, neoadjuvant PD-1 inhibitor nivolumab was administered to patients with surgically resectable, early-stage NSCLC (stage I, II, or IIIa) 4 weeks preoperatively. The primary endpoints of this trial were safety and feasibility. A total of 21 patients were included for treatment with neoadjuvant nivolumab followed by surgical resection of the tumour. In 20 patients, an $\mathrm{R} 0$ resection was achieved, and a major pathological response (MPR) occurred in $9 / 20$ resected tumours (45\%). Furthermore, results showed a recurrence rate of $73 \%$ within 18 months and an overall survival rate of $95 \%$. Treatment-related adverse events of any grade occurred in 5 patients, with one patient having a grade 3-4 adverse event (pneumonia). No treatment-related surgical delays were found in this study. The authors concluded that neoadjuvant nivolumab is a safe and feasible treatment with minimal side effects and favourable pathological response (41). In a similar trial, Bott et al. investigated the results of pulmonary resection after neoadjuvant nivolumab in patients with untreated stage I-IIIa NSCLC. A total of 20 patients underwent resection (15 lobectomies, 2 pneumonectomies, 1 bilobectomy, 1 sleeve lobectomy, and 1 wedge resection) via thoracoscopic surgery or thoracotomy. The authors found that nivolumab did not cause delays to surgical resection. There were no operative mortalities and rates of perioperative morbidities were minimal. In 9/20 patients (45\%), MPR was identified. However, 7/13 (54\%) procedures that were performed using thoracoscopic approaches had to be converted to thoracotomy, often due to hilar inflammation and fibrosis (42).

In the NEOSTAR phase II trial, nivolumab and nivolumab+ipilimumab were compared in 44 resectable NSCLC patients. The results showed a pathological complete response in 6 patients $(15 \%)$ and overall MPR rate of $24 \%$. Further subgroup analysis of 34 resected patients showed an MPR rate of $29 \%$. Postoperative complication rates were similar in both treatment arms; however, the nivolumab+ipilimumab arm showed higher rates of non-viable tumour. In the phase II, multi-centre NADIM trial, the efficacy of combining nivolumab with conventional chemotherapy was investigated. A total of 41 patients underwent surgical resection, with no delay due to neoadjuvant treatment. R0 resections were achieved in all patients. MPR was seen in $34 / 41$ patients $(83 \%)$ and a total of 24 patients $(71 \%)$ had a complete pathological response. The authors concluded that neoadjuvant chemotherapy combined with immunotherapy yields excellent pathological response rates (43). In addition to these trials, there are currently a number of ongoing randomized phase III trials regarding neoadjuvant immunotherapy in early-stage and (locally) advanced NSCLC. These are listed in Table 1.

Regarding targeted therapy, several studies have shown that EGFR-tyrosine kinase inhibitors (TKIs) and ALK inhibitors can significantly improve progression-free survival in specific subsets of patients with advanced stages of NSCLC (48-50). However, data from large prospective studies regarding the safety and efficacy of targeted therapies in a neoadjuvant setting are still lacking. A number of trials have been published in recent years regarding neoadjuvant targeted therapy. The EMERGING trial was a randomised phase II clinical trial that compared the safety and efficacy of erlotinib, an EGFR-TKI, with platinum-based doublet 
Table 1 Overview of ongoing randomized phase III trials regarding neoadjuvant immunotherapy

\begin{tabular}{|c|c|c|c|c|}
\hline NCT identifier (name) & Status & Intervention & $\begin{array}{c}\text { Estimated } \\
\text { enrolment }(\mathrm{N})\end{array}$ & $\begin{array}{l}\text { Primary } \\
\text { endpoint }\end{array}$ \\
\hline $\begin{array}{l}\text { NCT02998528 } \\
\text { (CheckMate 816) (44) }\end{array}$ & $\begin{array}{l}\text { Active, not } \\
\text { recruiting }\end{array}$ & $\begin{array}{l}\text { Arm A: } 3 \text { cycles nivolumab+ipilimumab } \rightarrow \text { Surgery } \rightarrow C T \pm R T \\
\text { Arm B: } 3 \text { cycles platinum doublet } \rightarrow \text { surgery } \rightarrow C T \pm R T \\
\text { Arm C: } 3 \text { cycles nivolumab+platinum doublet } \rightarrow \text { surgery } \rightarrow C T \pm R T\end{array}$ & 350 & EFS, pCR \\
\hline $\begin{array}{l}\text { NCT03425643 } \\
\text { (KEYNOTE-671) (46) }\end{array}$ & $\begin{array}{l}\text { Active, } \\
\text { recruiting }\end{array}$ & $\begin{array}{l}\text { Arm A: } 4 \text { cycles pembrolizumab+ platinum doublet } \rightarrow \text { surgery } \rightarrow \\
\text { pembrolizumab } \\
\text { Arm B: } 4 \text { cycles placebo+platinum doublet } \rightarrow \text { surgery } \rightarrow \text { placebo }\end{array}$ & 786 & EFS, OS \\
\hline
\end{tabular}

CT, chemotherapy; EFS, event-free survival; MPR, major pathological response; NCT, national clinical trial; OS, overall survival; pCR, pathologic complete response; RT, radiotherapy.

chemotherapy. The primary endpoint of this trial was the objective response rate (ORR) of the preoperative induction therapy. Although there was no significant difference in ORR, the ORR value of the erlotinib arm was objectively better than the chemotherapy arm $(54.1 \%$ vs. $34.3 \%$, respectively. The rate of R0 resections for the erlotinib and chemotherapy arm were $73 \%$ and $63 \%$, respectively. The progression-free survival was significantly better in the erlotinib group compared to the chemotherapy group (21.5 vs. 11.4 months, $\mathrm{P}<0.0001$ ). No significant difference was found regarding OS rates. Median overall survival of the erlotinib and chemotherapy groups were 45.8 and 39.2 months, respectively. Furthermore, a lower incidence rate of grade 3-4 adverse events was noted in the erlotinib group compared to the chemotherapy group (51). In the single-arm, single-centre, phase II ESTERN study, the safety and efficacy of erlotinib as neoadjuvant treatment for patients with IIIa-N2 NSCLC was evaluated. The primary endpoint of this study was the radical resection rate after 8 weeks of treatment. A total of 16 patients underwent lung resection and in $15 / 16$ patients $(93.8 \%)$, a complete resection was achieved (52).

Regarding outcomes of ALK inhibitors as neoadjuvant, only limited data is available. In a retrospective analysis of 11 ALK-positive patients with pathologically confirmed N2 NSCLC, neoadjuvant treatment with crizotinib resulted in a partial response in 10 patients (91\%). Furthermore, $\mathrm{R} 0$ resections were achieved in 10 patients and a complete pathological response was seen in 2 patients. Grade 4 hepatic damage was noted in 1 patient. Recurrent disease occurred in 6 patients, however, 5 patients showed a long duration of response to first-line crizotinib. The authors concluded that neoadjuvant crizotinib may be feasible and well tolerated in locally advanced NSCLC before complete resection. Furthermore, neoadjuvant crizotinib did not seem to influence response rates for the reuse of first-line crizotinib (53). Currently, there are a number of ongoing phase II trials regarding neoadjuvant targeted therapies investigating the efficacy of osimertinib (NCT03433469) and crizotinib (NCT03088930) in early-stage and locally advanced NSCLC $(54,55)$.

\section{Discussion}

Treatment for NSCLC has entered the era of precision medicine with new emerging therapies being introduced at an increasingly rapid pace. Although these new modalities seem promising, incorporating them in the current treatment approaches is still controversial (56). Trials regarding neoadjuvant targeted therapy and immunotherapy have shown that favourable outcomes can be achieved in early-stage and advanced stages of NSCLC (41-43,48-53). Furthermore, surgical outcomes after biological and immunotherapeutic treatments seem to be encouraging. However, the majority of data published regarding the combination of immunotherapy or targeted therapy and 
surgery have incorporated surgery immediately after systemic therapy. There is still very limited data regarding salvage surgery in long-term survivors after immunotherapy or targeted therapy.

In a case series of 5 patients, Chaft $e t$ al. presented their results of patients treated with lung resections after T-cell checkpoint inhibitor therapy for advanced stage NSCLC. They showed that lung cancer surgery is feasible and safe in long-term survivors after immunotherapy, even in patients who develop pneumonitis or other immunerelated toxicities during their treatment. The authors noted that dense fibrosis may develop in some patients as a result of excellent response to the systemic therapy, often making mediastinal and hilar dissection more technically challenging for thoracic surgeons (57). In another recent case series, Hamaji et al. described 2 patients that underwent salvage surgery after nivolumab administration. The salvage surgery was performed 9 and 12 months postnivolumab in these 2 patients. Both patients underwent video-assisted thoracoscopic resection of the lesions and had no postoperative complications. The authors concluded that delayed salvage surgery after nivolumab was feasible and was associated with little fibrosis in the hilar region intraoperatively (58).

Regarding salvage surgery after targeted therapy, Hishida $e t$ al. posted a case series of 9 patients that underwent surgical resection after treatment with gefitinib for advanced NSCLC. R0 resection was accomplished in all patients and no major postoperative complications were seen. However, 7/9 patients showed a more advanced pathological stage than their preoperative clinical stage. OS and DFS were 32 and 6 months, respectively. The authors commented that although salvage surgery was feasible and safe, postoperative survival outcomes were not satisfactory. They concluded that surgery after gefitinib should be limited to patients without initial evidence of disseminated and distant metastases (59). More recent data from limited case series have shown similar results for gefitinib and other EGFR-TKIs (60).

All these results seem to suggest that salvage surgery after immunotherapy and targeted therapy is feasible with favourable outcomes regarding local control in selected patient groups. However, the optimal timing and validity of salvage surgery for residual lesions after biologicals and immunotherapy remains unclear. More data is needed to define the optimal criteria for proceeding with lung resections in patients receiving these systemic therapies. Future clinical trials will hopefully provide answers to these questions and clarify which subset of patients will benefit from a multimodal approach combining these emerging therapies and (salvage) surgery.

\section{Acknowledgments}

Funding: None.

\section{Footnote}

Provenance and Peer Review: This article was commissioned by the Guest Editors (Lorenzo Spaggiari and Luca Bertolaccini) for the series "The Role of Pneumonectomy in Thoracic Surgery in The Third Millennium" published in Shanghai Chest. The article was sent for external peer review organized by the Guest Editors and the editorial office.

Conflicts of Interest: Both authors have completed the ICMJE uniform disclosure form (available at http:// dx.doi.org/10.21037/shc-2019-rpts-25). The series "The Role of Pneumonectomy in Thoracic Surgery in The Third Millennium" was commissioned by the editorial office without any funding or sponsorship. EPVS reports institutional fee as external expert from AstraZeneca, outside the submitted work. The authors have no other conflicts of interest to declare.

Ethical Statement: The authors are accountable for all aspects of the work in ensuring that questions related to the accuracy or integrity of any part of the work are appropriately investigated and resolved.

Open Access Statement: This is an Open Access article distributed in accordance with the Creative Commons Attribution-NonCommercial-NoDerivs 4.0 International License (CC BY-NC-ND 4.0), which permits the noncommercial replication and distribution of the article with the strict proviso that no changes or edits are made and the original work is properly cited (including links to both the formal publication through the relevant DOI and the license). See: https://creativecommons.org/licenses/by-nc-nd/4.0/.

\section{References}

1. Lu T, Yang X, Huang Y, et al. Trends in the incidence, treatment, and survival of patients with lung cancer in the last four decades. Cancer Manag Res 2019;11:943-53. 
2. Wong MCS, Lao XQ, Ho KF, et al. Incidence and mortality of lung cancer: global trends and association with socioeconomic status. Sci Rep 2017;7:14300.

3. de Groot PM, Wu CC, Carter BW, et al. The epidemiology of lung cancer. Transl Lung Cancer Res 2018;7:220-33.

4. Stewart BW, Wild CP. World cancer report 2014. 2014.

5. Siegel RL, Miller KD, Jemal A. Cancer statistics, 2020. CA Cancer J Clin 2020;70:7-30.

6. Zugazagoitia J, Molina-Pinelo S, Lopez-Rios F, et al. Biological therapies in nonsmall cell lung cancer. Eur Respir J 2017;49:1601520.

7. Lang-Lazdunski L. Surgery for nonsmall cell lung cancer. Eur Respir Rev 2013;22:382-404.

8. Ginsberg RJ, Rubinstein LV. Randomized trial of lobectomy versus limited resection for T1 N0 non-small cell lung cancer. Lung Cancer Study Group. Ann Thorac Surg 1995;60:615-22; discussion 22-3.

9. Fietkau R, Semrau S. Stage III: definitive chemoradiotherapy. Front Radiat Ther Oncol 2010;42:122-34.

10. Askoxylakis V, Tanner J, Kappes J, et al. Trimodal therapy for stage III-N2 non-small-cell lung carcinoma: a single center retrospective analysis. BMC Cancer 2014;14:572.

11. Evison M, Clive A, Castle L, et al. Resectable Clinical N2 Non-Small Cell Lung Cancer; What Is the Optimal Treatment Strategy? An Update by the British Thoracic Society Lung Cancer Specialist Advisory Group. J Thorac Oncol 2017;12:1434-41.

12. Auperin A, Le Pechoux C, Rolland E, et al. Meta-analysis of concomitant versus sequential radiochemotherapy in locally advanced non-small-cell lung cancer. J Clin Oncol 2010;28:2181-90.

13. Bradley JD, Paulus R, Komaki R, et al. Standard-dose versus high-dose conformal radiotherapy with concurrent and consolidation carboplatin plus paclitaxel with or without cetuximab for patients with stage IIIA or IIIB non-small-cell lung cancer (RTOG 0617): a randomised, two-by-two factorial phase 3 study. Lancet Oncol 2015;16:187-99.

14. Schreiner W, Dudek W, Lettmaier S, et al. Long-Term Survival after Salvage Surgery for Local Failure after Definitive Chemoradiation Therapy for Locally Advanced Non-small Cell Lung Cancer. Thorac Cardiovasc Surg 2018;66:135-41.

15. Mok TS, Wu YL, Thongprasert S, et al. Gefitinib or carboplatin-paclitaxel in pulmonary adenocarcinoma. N Engl J Med 2009;361:947-57.
16. Schreiner W, Dudek W, Lettmaier S, et al. Should salvage surgery be considered for local recurrence after definitive chemoradiation in locally advanced non-small cell lung cancer? J Cardiothorac Surg 2016;11:9.

17. Bauman JE, Mulligan MS, Martins RG, et al. Salvage lung resection after definitive radiation (>59 Gy) for non-small cell lung cancer: surgical and oncologic outcomes. Ann Thorac Surg 2008;86:1632-8; discussion 8-9.

18. Jeremic B, Videtic GM. Chest reirradiation with external beam radiotherapy for locally recurrent non-smallcell lung cancer: a review. Int J Radiat Oncol Biol Phys 2011;80:969-77.

19. McAvoy S, Ciura K, Wei C, et al. Definitive reirradiation for locoregionally recurrent non-small cell lung cancer with proton beam therapy or intensity modulated radiation therapy: predictors of high-grade toxicity and survival outcomes. Int J Radiat Oncol Biol Phys 2014;90:819-27.

20. Tetar S, Dahele M, Griffioen G, et al. High-dose conventional thoracic re-irradiation for lung cancer: updated results. Lung Cancer 2015;88:235-6.

21. Noble J, Ellis PM, Mackay JA, et al. Second-line or subsequent systemic therapy for recurrent or progressive non-small cell lung cancer: a systematic review and practice guideline. J Thorac Oncol 2006;1:1042-58.

22. Hanna N. Second-line treatment of non-small cell lung cancer: big targets, small progress; small targets, big progress? J Thorac Oncol 2006;1:927-8.

23. Paramanathan A, Solomon B, Collins M, et al. Patients treated with platinum-doublet chemotherapy for advanced non--small-cell lung cancer have inferior outcomes if previously treated with platinum-based chemoradiation. Clin Lung Cancer 2013;14:508-12.

24. Kaba E, Ozyurtkan MO, Ayalp K, et al. Salvage thoracic surgery in patients with lung cancer: potential indications and benefits. J Cardiothorac Surg 2018;13:13.

25. Casiraghi M, Maisonneuve P, Piperno G, et al. Salvage Surgery After Definitive Chemoradiotherapy for Nonsmall Cell Lung Cancer. Semin Thorac Cardiovasc Surg 2017;29:233-41.

26. Dickhoff C, Dahele M, Paul MA, et al. Salvage surgery for locoregional recurrence or persistent tumor after high dose chemoradiotherapy for locally advanced non-small cell lung cancer. Lung Cancer 2016;94:108-13.

27. Uramoto H, Tanaka F. Salvage thoracic surgery in patients with primary lung cancer. Lung Cancer 2014;84:151-5.

28. Uramoto H. Current Topics on Salvage Thoracic Surgery in Patients with Primary Lung Cancer. Ann Thorac Cardiovasc Surg 2016;22:65-8. 
29. Bertolaccini L, Spaggiari L. Salvage pneumonectomy after definitive chemo-radiotherapy. Shanghai Chest 2020;4:14.

30. Shiraishi T, Hiratsuka M, Yanagisawa J, et al. Pulmonary resection after chemoradiotherapy for advanced nonsmall cell lung cancer: the impact of presurgical radiation therapy. Surg Today 2014;44:123-30.

31. Yang CF, Meyerhoff RR, Stephens SJ, et al. Long-Term Outcomes of Lobectomy for Non-Small Cell Lung Cancer After Definitive Radiation Treatment. Ann Thorac Surg 2015;99:1914-20.

32. Sonobe M, Yutaka Y, Nakajima D, et al. Salvage Surgery After Chemotherapy or Chemoradiotherapy for Initially Unresectable Lung Carcinoma. Ann Thorac Surg 2019; 108:1664-70.

33. Dong J, Li B, Lin D, et al. Advances in Targeted Therapy and Immunotherapy for Non-small Cell Lung Cancer Based on Accurate Molecular Typing. Front Pharmacol 2019;10:230.

34. Bott MJ, Cools-Lartigue J, Tan KS, et al. Safety and Feasibility of Lung Resection After Immunotherapy for Metastatic or Unresectable Tumors. Ann Thorac Surg 2018;106:178-83.

35. Lu M, Su Y. Immunotherapy in non-small cell lung cancer: The past, the present, and the future. Thorac Cancer 2019;10:585-6.

36. Corrales L, Scilla K, Caglevic C, et al. Immunotherapy in Lung Cancer: A New Age in Cancer Treatment. Adv Exp Med Biol 2018;995:65-95.

37. Chan BA, Hughes BGM. Targeted therapy for non-small cell lung cancer: current standards and the promise of the future. Transl Lung Cancer Res 2015;4:36-54.

38. Sa H, Song P, Ma K, et al. Perioperative Targeted Therapy Or Immunotherapy In Non-Small-Cell Lung Cancer. Onco Targets Ther 2019;12:8151-9.

39. De Velasco G, Je Y, Bossé D, et al. Comprehensive Metaanalysis of Key Immune-Related Adverse Events from CTLA-4 and PD-1/PD-L1 Inhibitors in Cancer Patients. Cancer Immunol Res 2017;5:312-8.

40. Abdel-Wahab N, Shah M, Suarez-Almazor ME. Adverse Events Associated with Immune Checkpoint Blockade in Patients with Cancer: A Systematic Review of Case Reports. PLoS One 2016;11:e0160221.

41. Forde PM, Chaft JE, Smith KN, et al. Neoadjuvant PD-1 Blockade in Resectable Lung Cancer. N Engl J Med 2018;378:1976-86.

42. Bott MJ, Yang SC, Park BJ, et al. Initial results of pulmonary resection after neoadjuvant nivolumab in patients with resectable non-small cell lung cancer. J
Thorac Cardiovasc Surg 2019;158:269-76.

43. Cascone T, William WN, Weissferdt A, et al. Neoadjuvant nivolumab $(\mathrm{N})$ or nivolumab plus ipilimumab $(\mathrm{NI})$ for resectable non-small cell lung cancer (NSCLC): Clinical and correlative results from the NEOSTAR study. J Clin Oncol 2019;37:8504.

44. ClinicalTrials.gov. A Neoadjuvant Study of Nivolumab Plus Ipilimumab or Nivolumab Plus Chemotherapy Versus Chemotherapy Alone in Early Stage Non-Small Cell Lung Cancer (NSCLC). Available online: https://ClinicalTrials. gov/show/NCT02998528

45. ClinicalTrials.gov. A Study of Neoadjuvant Atezolizumab Plus Chemotherapy Versus Placebo Plus Chemotherapy in Patients With Resectable Stage II, IIIA, or Select IIIB Non-Small Cell Lung Cancer (IMpower030). Available online: https://ClinicalTrials.gov/show/NCT03456063

46. ClinicalTrials.gov. Efficacy and Safety of Pembrolizumab (MK-3475) With Platinum Doublet Chemotherapy as Neoadjuvant/Adjuvant Therapy for Participants With Resectable Stage II, IIIA, and Resectable IIIB (T34N2) Non-small Cell Lung Cancer (MK-3475-671/ KEYNOTE-671). Available online: https://ClinicalTrials. gov/show/NCT03425643

47. ClinicalTrials.gov. A Study of Neoadjuvant Chemotherapy Plus Nivolumab Versus Neoadjuvant Chemotherapy Plus Placebo, Followed by Surgical Removal and Adjuvant Treatment With Nivolumab or Placebo for Participants With Surgically Removable Early Stage Non-small Cell Lung Cancer. Available online: https://ClinicalTrials.gov/ show/NCT04025879

48. Sellmann L, Fenchel K, Dempke WC. Improved overall survival following tyrosine kinase inhibitor treatment in advanced or metastatic non-small-cell lung cancer-the Holy Grail in cancer treatment? Transl Lung Cancer Res 2015;4:223-7.

49. Karachaliou N, Rosell R. Systemic treatment in EGFRALK NSCLC patients: second line therapy and beyond. Cancer Biol Med 2014;11:173-81.

50. Elliott J, Bai Z, Hsieh SC, et al. ALK inhibitors for nonsmall cell lung cancer: A systematic review and network meta-analysis. PLoS One 2020;15:e0229179.

51. Zhong WZ, Chen KN, Chen C, et al. Erlotinib Versus Gemcitabine Plus Cisplatin as Neoadjuvant Treatment of Stage IIIA-N2 EGFR-Mutant Non-Small-Cell Lung Cancer (EMERGING-CTONG 1103): A Randomized Phase II Study. J Clin Oncol 2019;37:2235-45.

52. Han B, Xiong L, Sun J, et al. Erlotinib as Neoadjuvant Treatment in Endobronchial Ultrasound Confirmed 
Stage IIIA-N2 Non-Small Cell Lung Cancer (NSCLC) Patients with Epidermal Growth Factor Receptor (EGFR) Mutation (EXON 19 OR 21) (NCT01217619, ESTERN): a Prospective, Single Arm, Phase Ii Clinical Trial. Ann Oncol 2015;26:i24.

53. Zhang C, Li SL, Nie Q, et al. Neoadjuvant Crizotinib in Resectable Locally Advanced Non-Small Cell Lung Cancer with ALK Rearrangement. J Thorac Oncol 2019;14:726-31.

54. ClinicalTrials.gov. Osimertinib in Treating Participants With Stage I-IIIA EGFR-mutant Non-small Cell Lung Cancer Before Surgery. Available online: https:// ClinicalTrials.gov/show/NCT03433469

55. ClinicalTrials.gov. Evaluating Crizotinib in the Neoadjuvant Setting in Patients With Non-small Cell Lung Cancer. Available online: https://ClinicalTrials.gov/ show/NCT03088930

doi: 10.21037/shc-2019-rpts-25

Cite this article as: Berzenji L, Van Schil PE. Salvage lung surgery in long-term survivors after biological and immunotherapeutic treatments. Shanghai Chest 2021;5:24.
56. Jones CM, Brunelli A, Callister ME, et al. Multimodality Treatment of Advanced Non-small Cell Lung Cancer: Where are we with the Evidence? Curr Surg Rep 2018;6:5.

57. Chaft JE, Hellmann MD, Velez MJ, et al. Initial Experience With Lung Cancer Resection After Treatment With T-Cell Checkpoint Inhibitors. Ann Thorac Surg 2017;104:e217-8.

58. Hamaji M, Ozasa H, Yoshizawa A, et al. Salvage thoracoscopic resection after nivolumab for stage IV. Asian Cardiovasc Thorac Ann 2020;28:216-8.

59. Hishida T, Nagai K, Mitsudomi T, et al. Salvage surgery for advanced non-small cell lung cancer after response to gefitinib. J Thorac Cardiovasc Surg 2010;140:e69-71.

60. Yamamoto Y, Kodama K, Maniwa T, et al. Surgical resection of advanced non-small cell lung cancer after a response to EGFR-TKI: presentation of two cases and a literature review. J Cardiothorac Surg 2017;12:98. 